\title{
Características morfológicas y patogénicas de aislamientos de Septoria tritici Rob ex Desm
}

\author{
A Perelló, CA Cordo, HE Alippi \\ Comisión de investigaciones cientificas de la provincia de Buenos Aires (CIC), Universidad Nacional de La Plata, \\ Cátedra de Fitopatología de la Facultad de Agronomia, Calle 60 y 118 (1900) La Plata, Pcia, de Bs Aires, Argentina
}

(Recibido el 6 de octubre del 1989; admitido el 12 de julio del 1990)

Resumen - Se analizó el comportamiento cultural y patogénicos de 8 aislamientos de Septoria tritici obtenidos a partir de hojas de trigo colectadas en el campo e infectadas naturalmente.

Los diversos aislamientos monospóricos pueden diferenciarse en cultivo artificial por su aspecto, la producción de esporas, la presencia o ausencia de micelio, y el color y la frecuencia de saltaciones observadas en las colonias.

Las cepas varían tanto en la abundancia como en el tipo de las esporas producidas bajo condiciones similares. Además de las picnidiosporas de forma y tamaño típicos de la especie, algunas cepas produjeron sólo esporas libres muy pequeñas (microsporas), y otras, macro y microsporas dentro des picnidios.

Los tipos culturales predominantes de los aislamientos fueron 5 :

- estromático (mayor frecuencia),

- albino pulverulento,

- levaduroide,

- mixto y

- miceliar.

Estos tipos culturales, en algunos casos no se mantuvieron estables a través de 3 generaciones de subcultivos.

Las inoculaciones efectuadas sobre plantas de trigo en invernáculo, revelaron diferencias altamente significativas en la patogenicidad de ciertas cepas. Se encontró una relación entre las características culturales de los aislamientos y la severidad de la enfermedad: los aislamientos de tipo albino y levaduroide fueron los menos virulentos, tanto sobre variedades resistentes como susceptibles.

La presente, constitutíye la primer cita de la presencia de esta clase de microsporas de $S$ tritici.

\section{microspora / septoria tritici / aislamiento}

Summary - Morphological and pathogenic characteristics of isolates of Septoria tritici Rob Ex Desm. This study analyzed the cultural and pathogenic behavior of $S$ tritici isolates from wheat leaves infected and collected in the field.

When cultured artificially, monosporic isolates were differenciated in aspect, spore production, presence or absence of mycelium, and in color and frequency of saltation observed in the colonies as shown in table I. The characteristics noted led to the classification of the cultures as mycelial (M), typical (stromatic-pycnidial) (T) (the most frequent), powdery albino (A), yeast-like ( $Y$ ) and mixed (MX) (figs 1 and 2). In some cases these cultural types, though pure, did not remain stable after three subculture generations.

The variants from the parental type appeared patches or sectorings of the yeast-like and/or the mycelial type. Sometimes these appeared in the first generation, some others in the third generation, with a low frequency of appearance with respect to the parental type.

In the greenhouse, inoculation in the form of wheat plants revealed highly significant differences in the pathogenicity of some strains. Correlation of the mean percentages of pycnidial coverage showed a significant difference in isolate pathogenicity levels in the same cultivar and within cultivars (table II). Thus, the existence of physiologic specialization in $\mathrm{S}$ tritici is again confirmed. A relationship was found between the isolates' cultural characteristics and the disease severity: the albino and yeast-like isolates were the least virulent for both the resistant and susceptible cultivars.

Under similar conditions the strains varied in spore abundance as in the spore type produced (fig 4). Besides pycnidiospores with shape and size characteristics of the species, some strains produced only very small free spores (microspores), and some others macro and microspores within pycnidia (table III). Microspores produced in vivo were much smaller than those obtained artificially. This is the first report regarding this type of microspore in $\mathrm{S}$ tritici. 


\section{INTRODUCCIÓN}

Septoria tritici produce macropicnidiosporas predominantes y típicas de la especie y micropicnidiosporas (Dickson, 1947, Sprague, 1950). Estas últimas no son frecuentes en condiciones naturales y se presentan sólas o en asociación con las macropicnidiosporas (Sprague, 1950). Se ha sugerido (Brokenshire, 1975) que ellas no actúan como inóculo, y que funcionarían como espermas en el proceso sexual.

Por otra parte, $S$ tritici produce conidios secundarios in vitro (Djerbi, 1972; Jones and Lee, 1974) y recientemente se confirmó y documentó su ocurencia in vivo (Annone, 1988).

En cultivo artificial, el patógeno desarrolla colonias con distintos hábitos de crecimiento (Annone, 1984), siendo el más frecuente el conidial (levaduroide). Para nuestro país, hasta el presente, se encontraron los tipos picnidial y miceliar, con predominancia del primero (Cordo $y$ Lindquist, 1987).

En $S$ nodorum, Skajennikoff y Rapilly (1983, 1989) observaron in vitro diferencias morfológicas entre los diversos aislamientos. Así mismo, comprobaron que varian su agresividad, después de pasajes a través de ciertos hospedantes que ejercen una presión de seleccion sobre la población del patógeno. En Argentina, actualmente, se están estudiando aislamiento de $S$ tritici provenientes de cultivares de trigo con germoplasma extranjero (Bobwhite "S") cuyos caracteres culturales, morfobiométricos y de virulencia fueron distintos a los obtenidos a partir de cultivares nacionales.

Debido a que el conocimiento de la variacíon cultural y del tipo y tamaño de las esporas puede ser importante en estudios taxonómicos y epidemiológicos, se inició esta investigación preliminar. El objetivo fué caracterizar las variantes culturales del patógeno y comparar sus virulencias. También se intentó determinar la relación existentes entre ciertas características culturales y la patogenicidad de $S$ tritici.

\section{MATERIALES Y MÉTODOS}

\section{Variabilidad cultural}

Se estudió la variabilidad cultural de 3 aislamiento monospóricos representativos y típicos de $S$ tritici: $B_{18}, D_{11}$ y $L H_{5}$. Se seleccionaron desde una amplia coleccion del patógeno, obtenida a partir de cultivares de trigo nacionales que se hallaban infectados naturalmente. Se les aisló mediante el método directo (Eyal et al, 1987). Estos aislamientos, excepto $\mathrm{LH}_{5}$, fueron estables luego de repicarse periódicamente y de mantenerse en tubos con el medio Czapek Dox $V_{8}$ (Plant Pathologist's Pocketbook, 1968) en cámara refrigerante a $6-10^{\circ} \mathrm{C}$ por $6 \mathrm{~m}-1 \mathrm{a}$. Se incluyó también en este estudio, un aislamiento de tipo mixto: $P_{6}$, uno miceliar: $M_{16}$ y un mutante albino: $\mathrm{LH}_{5} \mathrm{~m}$ aparecido en cultivo a partir del aislamiento $\mathrm{LH}_{5}$, luego de varias generaciones de sub-aislamientos monospóricos.

Por último, se analizaron dos aislamientos: $P_{1}$ y $P_{3}$ obtenidos desde plantas de trigo de un cultivar extranjero con germoplasma resistente (tabla I).

Se cultivaron durante $4 \mathrm{~s}$ en cajas de Petri y tubos con el medio Agar Papa Glucosado (APG), bajo condiciones de laboratorio (temperatura media de $20^{\circ} \mathrm{C}$ y luz difusa). Finalizado este tiempo, se observó el aspecto de la colonia, color (según Rayner, 1970), superficie, forma, borde, estructura interna y dimensiones y tipos de esporas de los aislamientos. Para la descripción de las colonias, se siguió a Garassini (1958) y Negroni (1938).

De cada línea original, se estudiaron tres generaciones por transferencias monospóricas $\left(G_{1}, G_{2}\right.$ y $\left.G_{3}\right)$ en tubos de ensayo con Agar Malta (AM) ( $30 \mathrm{~g}$ de extracto de malta, $5 \mathrm{~g}$ de peptonas micológicas, $20 \mathrm{~g}$ de agar y $1000 \mathrm{ml}$ de agua destilada) y APG. Para iniciar cada generación, se consideró solamente el tipo cultural predominante en la colonia, cuando esta presentó alguna sectorización. Las variantes (zonaciones o sectorizaciones) aparecidas en una misma generacíon o entre generaciones, sólo se consideraron en cuanto a frecuencia de aparición (porcentaje).

\section{Variabilidad patogénica}

Se analizó la patogenicidad de los 8 aislamientos señalados. Se emplearon los cultivos monospóricos de $30 \mathrm{~d}$ desarrollados en tubos con el medio AM bajo las condiciones ya indicadas.

Se preparó una suspención de esporas y/o esporas y micelio en $250 \mathrm{ml}$ de agua destilada estéril. La suspensión inoculante se ajustó a $1 \times 10^{7}$ unidades infectivas $/ \mathrm{ml}$. Se inocularon plantas de trigo con $3 \mathrm{~h}$ desarrolladas de una variedad resistente (Bordenave Puán) y de una variedad susceptible (Marcos Juarez INTA) a $S$ tritici.

Se empleó un diseño completamente al azar con 5 repeticiones, considerando a cada maceta con 5 plantas como una repetición. Las plantas se mantuvieron bajo condiciones controladas de invernáculo (temperatura media de $21^{\circ} \mathrm{C}$ y humedad relativa de $75 \%$ ).

La evaluación se realizó a los 28 d de la inoculación registrando el porcentaje de cubertura picnidial en cada tratamiento como se describió previamente (Perelló et al, 1987). 
Tabla I. Caracteristicas culturales de los 8 aislamiento de $S$ tritici observados a los 30 dias de cultivo en APG.

\begin{tabular}{|c|c|c|c|c|c|c|c|c|c|c|}
\hline \multicolumn{2}{|c|}{$\begin{array}{l}\text { Aslamiento } \\
\text { (codigo) }\end{array}$} & \multirow{2}{*}{$\begin{array}{c}\begin{array}{c}\text { Tipos } \\
\text { cultu- } \\
\text { rales }\end{array} \\
\text { Típica }\end{array}$} & \multirow{2}{*}{$\begin{array}{c}\text { Aspecto } \\
\text { (1) } \\
\text { E }\end{array}$} & \multirow{2}{*}{$\begin{array}{l}\text { Color } \\
\\
\text { Gris } \\
\text { oscuro }\end{array}$} & \multirow{2}{*}{$\begin{array}{c}\text { Super- } \\
\text { ficie } \\
(2) \\
\mathrm{L}\end{array}$} & \multirow{2}{*}{$\begin{array}{c}\text { For- } \\
\text { ma } \\
\text { (3) } \\
\mathrm{C}\end{array}$} & \multirow{2}{*}{$\begin{array}{l}\text { Bor- } \\
\text { de } \\
\text { (4) } \\
\text { F }\end{array}$} & \multirow{2}{*}{$\begin{array}{c}\begin{array}{c}\text { Estructura } \\
\text { interna }\end{array} \\
\text { Opaca }\end{array}$} & \multirow{2}{*}{$\begin{array}{c}\begin{array}{c}\text { Espo- } \\
\text { rulacion } \\
(5)\end{array} \\
\text { A }\end{array}$} & \multirow{2}{*}{\begin{tabular}{l}
\multicolumn{1}{c}{$\begin{array}{c}\text { Otras } \\
\text { obervaciones }\end{array}$} \\
$\begin{array}{l}\text { Micelio aéreo } \\
\text { algodonoso } \\
\text { gris claro, } \\
\text { blanco }\end{array}$
\end{tabular}} \\
\hline LH5 & $\begin{array}{l}\text { Línea no identificada } \\
\text { ECR de Los Hornos, } \\
\text { Prov Bs As }\end{array}$ & & & & & & & & & \\
\hline B18 & $\begin{array}{l}\text { Línea de Bobwhite } \\
\text { "S"PF 70354/Bob } \\
\text { white "S", Balcarce }\end{array}$ & Tipica & $E$ & $\begin{array}{l}\text { Gris } \\
\text { oscuro }\end{array}$ & $R u$ & $c$ & $E$ & $\begin{array}{l}\text { Opaca } \\
\text { Transpa- } \\
\text { rente }\end{array}$ & A & $\begin{array}{l}\text { Micelio aéreo } \\
\text { algodonoso } \\
\text { blanco }\end{array}$ \\
\hline LH5m & $\begin{array}{l}\text { Mutante } \\
\text { de } \\
\text { LH5 }\end{array}$ & $\begin{array}{l}\text { Mutante } \\
\text { albino }\end{array}$ & Y-P & Blanco & $\begin{array}{l}\mathrm{Ru} \\
\mathrm{Ra}\end{array}$ & $\begin{array}{l}\mathrm{C} \\
\mathrm{F}\end{array}$ & $\begin{array}{l}\mathrm{O} \\
\mathrm{F}\end{array}$ & $\begin{array}{l}\text { Tosca- } \\
\text { mente } \\
\text { Granulada }\end{array}$ & a & $\begin{array}{l}\text { Desarrollo } \\
\text { trifugo Secto- } \\
\text { rizaciones }\end{array}$ \\
\hline M16 & $\begin{array}{l}\text { Cultivar Buck } \\
\text { Pucará } \\
\text { Pdo de Magdalena }\end{array}$ & Miceliar & $\mathrm{Mi}$ & $\begin{array}{l}\text { Gris } \\
\text { claro } \\
\text { Gris } \\
\text { oscuro }\end{array}$ & $\begin{array}{l}\mathrm{E} 1 \\
\mathrm{~F}\end{array}$ & $C$ & $E$ & Opaca & $P$ & $\begin{array}{l}\text { Micelio aéreo } \\
\text { blanco, algodo- } \\
\text { noso denso }\end{array}$ \\
\hline D11 & $\begin{array}{l}\text { Cultivar Buck } \\
\text { Napostá } \\
\text { Cuel Dorrego }\end{array}$ & Típica & $E$ & $\begin{array}{l}\text { Gris } \\
\text { oscuro }\end{array}$ & $R u$ & $\mathrm{C}$ & $E$ & Opaca & A & $\begin{array}{l}\text { Micelio aéreo } \\
\text { blanco } \\
\text { algodonoso }\end{array}$ \\
\hline P6 & $\begin{array}{l}\text { Línea de Bobwhite } \\
\text { "3"/Toro 23/86 } \\
\text { Pergamino }\end{array}$ & $\begin{array}{l}\text { Mu } \\
\text { Mixta }\end{array}$ & $\begin{array}{l}\mathrm{E} \\
\mathrm{Mi}\end{array}$ & $\begin{array}{l}\text { Rosado } \\
\text { gris claro } \\
\text { Gris } \\
\text { vedoso o }\end{array}$ & oscuro $L$ & C & $E$ & Opaca & A & $\begin{array}{l}3 \text { sectores } \\
\text { "miceliar } \\
\text { "conidial } \\
\text { "estromático }\end{array}$ \\
\hline P1 & $\begin{array}{l}\text { Línea de Bobwhite } \\
\text { "S"Bod/4/LPI } \\
\text { "S"/3/NAD//Bd } \\
\text { IHTAZ LAS } 3139 \\
\text { Pergamino }\end{array}$ & Albina & $\begin{array}{l}\mathrm{Mu} \\
\mathrm{A}-\mathrm{B}\end{array}$ & $\begin{array}{l}\text { Blanco } \\
\text { amaril- } \\
\text { lento }\end{array}$ & $\begin{array}{l}\mathrm{E} \\
\mathrm{Ru}\end{array}$ & $C$ & $F$ & $\begin{array}{l}\text { Concén- } \\
\text { trica } \\
\text { enrulada }\end{array}$ & A & $\begin{array}{l}\text { Desarollo } \\
\text { "cerebroide" }\end{array}$ \\
\hline P3 & $\begin{array}{l}\text { Línea de Bobwhite } \\
\text { "S" LPI/Bob "S" }\end{array}$ & $\begin{array}{l}\text { Leva- } \\
\text { duroide } \\
\text { Rosada }\end{array}$ & $\begin{array}{l}\mathrm{Mu} \\
\mathrm{A}-\mathrm{B}\end{array}$ & Rosado & $L$ & $C$ & $F$ & Concentric & & $\begin{array}{l}\text { Sectori- } \\
\text { zaciones } \\
\text { en "V" }\end{array}$ \\
\hline
\end{tabular}

(1) $\mathrm{E}=$ Estromático; $\mathrm{Y}-\mathrm{P}=$ Yesoso-pulverulento; $\mathrm{Mi}=$ Miceliar; $\mathrm{Mu}=$ Mucaso; $\mathrm{A}-\mathrm{B}=$ Aterciopelado brillante. (2) $\mathrm{L}=\mathrm{Lisa}$ : Ru $=\mathrm{Ru}$ gosa; $R a=$ Radiada; $E I=$ Elevada. (3) $C=$ Circular; $F=$ Filamentosa. (4) $F=$ Filamentoso; $E=E n t e r o ; ~ O=O$ Ondulado. (5) $P=$ Pobre; A = Abundante. Caracteristicas de las colonias según Garassini (1958), Negroni y Rayner (1970). 


\section{Tipos y dimensiones de conidios in vitro}

Se determinó el tipo y dimensiones de los conidios de los 8 aislamientos analizados. Se tomó material de la periferia, centro y margen de cada colonia. Se montó en azul de algodón y lactofenol y luego se observó microscopicamente. Los conidios fueron medidos con un aumento de $400 \mathrm{x}$.

\section{Tipos y dimensiones de conidios in vivo}

Se observó el contenido de los picnidios desarrollados en las lesiones de hojas del material inoculado, mediante aplastado de los mismos. Los tipos de conidios hallados en las fructificaciones fueron medidos y morfologicamente descriptos.

\section{RESULTADOS}

\section{Variabilidad cultural}

Se encontraron diferencias culturales entre los 8 aislamientos analizados. Los caracteres más pobresalientes (aspecto, color, superficie, forma, borde, estructura interna y esporulación) de las colonias, se presentan en la tabla I.

Las características fenotípicas observadas, condujeron a clasificar a los cultivos como miceliar (M); típico (estromático-picnidial) (T); albino $(A)$; levaduroide (L) y mixto $\left(M_{x}\right)$ (fig 1 y 2 ).

\section{Típico}

Correspondió a los aislamientos $\mathrm{LH}_{5}, \mathrm{~B}_{18}$ y $\mathrm{D}_{11}$ (fig $1 \mathrm{~d}$ y fig $2 \mathrm{~b}$, d). Presenta une colonia es-

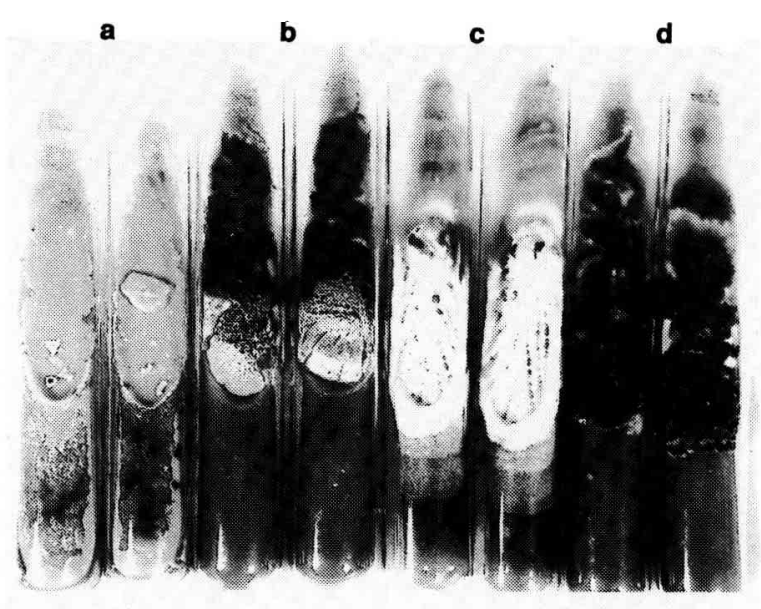

Fig 1. Tipos culturales de $S$ tritici desarrollados a los 30 días en APG (cada tipo está representado por 2 tubos de ensayo). $a=P_{3}$ (levaduroide rosado); $b=P_{6}$ (mixto); $c=M_{16}$ (miceliar) y d $=D_{11}$ (típico) tromática, constituída por un estroma gris oscuro (122. Iron Grey y 107, Grey olivaceous) de picnidios con cirros blancos de picnidiosporas en superficie. Presencia de micelio superficial blanco, algodonoso y compacto. En algunos casos la colonia presenta crecimiento radiado, constituído por anillos gris claro a gris oscuro (121. Olivaceous y 105. Smoke Grey respectivamente). La zona marginal está constituída por micelio aéreo gris claro (121. Olivaceous Grey) o por una masa brillosa, rosada, de conidios secundarios, de superficie lisa y deborde entero.

\section{Albino}

Se incluyeron dentro de esta clasificación a los aislamientos $L_{5} \mathrm{~m}_{\text {y }} \mathrm{P}_{1}$ (fig $2 \mathrm{a}, \mathrm{c}$ y fig $3 \mathrm{a}, \mathrm{c}$ ). $\mathrm{LH}_{5} \mathrm{~m}$, es una verdadera mutante y no el resultado de un cultivo mezclado. Esto lo refleja el hecho de que el cultivo parental $\left(\mathrm{LH}_{5}\right)$, ha sido repicado de 25 a 30 veces y desarrollado sobre varios medios y sobre un amplio rango de condiciones ambientales durante un período de 5 años sin que ocurrieran cambios.

El tipo albino, presenta una colonia mucosoaterciopelada 6 de aspecto yesoso-pulverulento y opaca. Esta zona está constituída por conidios secundarios y microsporas de color rosado (45. Buff). La zona marginal está constituída por micelio aéreo velutino, de desarrollo concéntrico, color 45. Buff o blanco o bien presentó un desarrollo radiado o curvo (con giro contrario a las agujas del reloj) (fig 3a). Se observaron sectorizaciones en forma de " $\mathrm{V}$ ", con zonaciones de aspecto afieltrado, superficie plana, lisa, color 117. Pale Mouse Grey, y micelio aéreo afieltrado laxo.

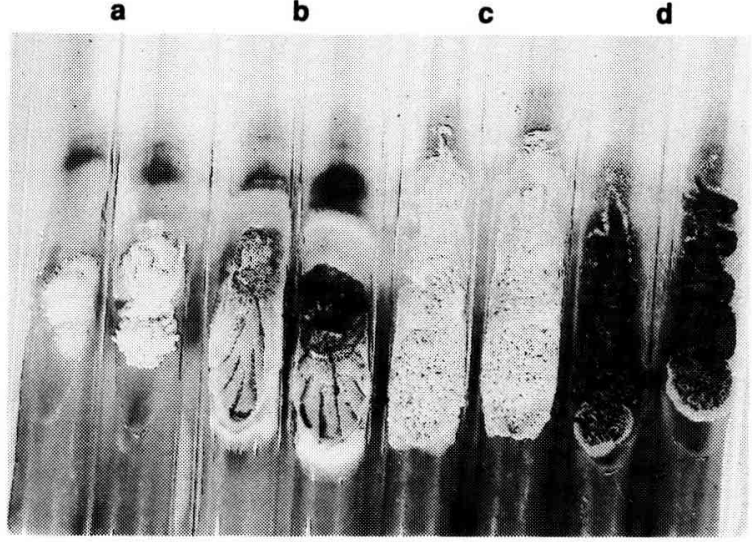

Fig 2. Tipos culturales de $S$ tritici desarrollados a los 30 dias en APG (cada tipo está representado por 2 tubos de ensayo). $\mathrm{a}=\mathrm{LH}_{5} \mathrm{~m}=$ (mutante albino); $\mathrm{LH}_{5}$ (típico); $\mathrm{c}=\mathrm{P}_{1}$ (albino) y $d=B_{18}$ (típico). 


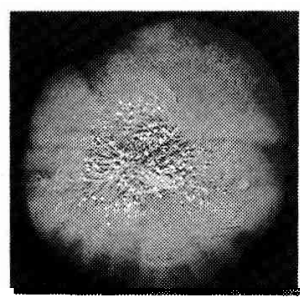

a

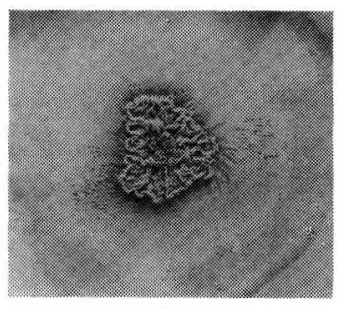

6

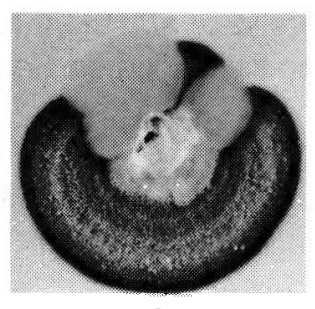

b

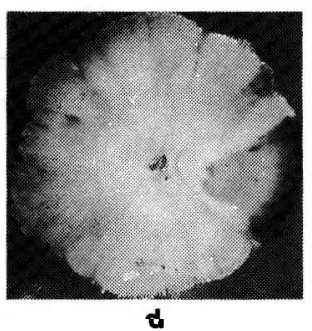

Fig 3. Detalle de los tipos culturales de $S$ tritici desarrollados en caja de Petri con APG a los 30 días. $a=\mathrm{LH}_{5} \mathrm{~m}$ (mutante albino); $b=P_{6}$ (mixto); $c=P_{1}$ (albino) y $d=P_{3}$ (levaduroide rosado).

\section{Miceliar}

Dentro de este tipo cultural, se incluyó al aislamiento $M_{16}$ (fig 1c). Las formas miceliares se observan muchas veces en la coleccion de cepas de $S$ tritici por envejecimiento del cultivo parental o por la presíon de selección ejercida luego de numerosos repiques. Sin embargo, la cepa $M_{16}$ fue encontrada naturalmente como una variante cultural miceliar de baja esporulacíon.

\section{Mixto}

Este tipo cultural corresponde al aislamiento $\mathrm{P}_{6}$ (fig $1 \mathrm{~b}$ y $3 \mathrm{~b}$ ). Presenta una colonia constituida por 3 zonas:

- zona de aspecto levaduroide, mucosa, brillante, constituida por conidios secundarios libres color 64. Rosy Buff; borde filamentoso y superficie lisa

- zona miceliar constituída por micelio blanco, algodonoso y compacto

- zona estromático-picnidial constituída por micelio verde grisáceo oscuro (122. Iron Grey y 107. Grey olivaceous); parte de este micelio se entremezcla con un estroma compacto, pardusco oscuro, de donde emergen cirros blanco-crema opacos constituídos por picnidiosporas. El micelio de esta zona se dispone en anillos concéntricos anchos, alternando el gris claro con el gris oscuro.

\section{Levaduroide}

Pertenece a este tipo el aislamiento $P_{3}$ (fig 1a y $3 d$ ). Presenta una colonia de aspecto levaduroide, mucoso, húmedo y brillante, color rosado (58. Rosy vinaceous); en la zona marginal se observa micelio aéreo de escasa densidad y desarrollo. Superficie con anillos concéntricos muy próximos constituidos por micelio aéreo rasante. Se observan sectorizaciones en " $\mathrm{V}$ ", constituídas por micelio radiado color verde oliváceo (107. Grey Olivaceous).

A través del estudio generacional, se concluye que 3 de los 8 aislamientos observados no se mantuvieron estables. Las variantes respecto al tipo parental predominante, aparecieron como parches o sectorizaciones del cultivo con aspecto levaduroide $y / 0$ miceliar. Estas variantes aparecieron en los aislamientos $L_{5} \mathrm{~m}, \mathrm{P}_{6}$ y $\mathrm{P}_{3}$. En algunos casos se detectaron en la primera generación, $y$ en otros en la tercera, manteniendo una frecuencia de aparición baja respecto al tipo parental. En el aislamiento $\mathrm{LH}_{5} \mathrm{~m}$ el $30 \%$ presentó zonaciones de tipo miceliar en la segunda generación. En el aislamiento $P_{6}$, se observaron un $10 \%$ de variantes de tipo levaduroide y miceliar respectivamente, en la primera generación, y un 10 y un $20 \%$ de cultivos con sectores miceliares en la segunda y tercera generación, respectivamente. En el aislamiento $P_{3}$, el $10 \%$ de los cultivos observados presentaron sectorizaciones de tipo miceliar en la segunda generación de transferencia.

Los aislamientos $L_{5} m, B_{18}, M_{16}, D_{11}$ y $P_{1}$ se mantuvieron estables.

\section{Variabilidad patogénica}

El análisis de varianza, reveló diferencias significativas, al nivel del $1 \%$ de probabilidad, entre los aislamientos para ambos cultivares. Las medias del porcentaje de cobertura picnidial inducidas por los 8 aislamientos en los 2 hospedantes probados, se presentan en la tabla II. La comparación de las medias a través del Test de Tukey, reveló que los aislamientos presentan diferentes niveles de patogenicidad. El aislamiento $\mathrm{LH}_{5} \mathrm{~m}$ fue el más agresivo sobre el cultivar Bordenave Puán, y los aislamientos $D_{11}$ y $B_{18}$ lo fueron sobre Marcos Juarez INTA. Otros dos aislamientos, $P_{1}$ y $P_{3}$, difieren significativamente del resto, por su menor agresividad para los 2 
Tabla II. Patogenicidad de los 8 aislamiento $S$ tritici sobre los cultivares de trigo Bordenave Puán y Marcos J INTA. Observaciones: Para la misma columna, las medias que poseden letras en común, no difieren estadísticamente $(P=0,01)$.

\begin{tabular}{|c|c|c|}
\hline \multirow{2}{*}{ Aislamiento } & \multicolumn{2}{|c|}{ Hospendantes } \\
\hline & Bordenave Puan & $\begin{array}{c}\text { Marcos Juarez } \\
\text { INTA }\end{array}$ \\
\hline LH5 & $31,68 a^{*}$ & $25,72^{d *}$ \\
\hline B18 & $20,16^{b}$ & $53,48^{a}$ \\
\hline LH5m & $19,64 b c$ & $24,88^{d}$ \\
\hline M16 & 17,02 bcd & $39,84^{c}$ \\
\hline D11 & 19,62 bcde & 55,38 a \\
\hline P6 & 15,60 cdef & 55,38 a \\
\hline P1 & 15,40 cdefg & $15,56 e^{e}$ \\
\hline P3 & 11,52 defg & $11,86^{e}$ \\
\hline DMS (Tuckey) ${ }^{\star \star} 1 \%$ & 4,48 & 5,36 \\
\hline CV & $9,85 \%$ & $6,34 \%$ \\
\hline
\end{tabular}

* Datos promedio de 25 plantas dados en porcentaje de cobertura picnidial.

cultivares analizados. En la variedad Bordenave Puán, se puede distinguir además, un tercer grupo en cuanto a virulencia, constituído por los aislamientos $B_{18}, L_{5} m, M_{16}, D_{11}$ y $P_{6}$, que, si bien se interrelacionan entre si, aparecen con un nivel de patogenicidad intermedio entre $\mathrm{LH}_{5}$ y $\mathrm{P}_{1}-$ $P_{3}$. En la variedad Marcos Juarez INTA, el nivel de patogenicidad intermedio está constituído por los aislamientos $P_{6}, M_{16}, L_{5}$ y $L_{5} m$, aunque entre ellos existieron diferencias significativas de comportamiento.

De la relación entre los tipos culturales definidos y la patogenicidad, surge que el típico fue el más virulento (indicado por la letra a), tanto sobre la variedad resistente como sobre la variedad susceptible. Por otra parte, los tipos culturales albino $\left(P_{1}\right)$ y levaduroide $\left(P_{3}\right)$ fueron los que demostraron la menor virulencia en ambos cultivares (indicado por las letras $\mathrm{g}$ y e respectivamente).

Estos resultados demuestran la existencia de una variación significativa en los niveles de patogenicidad de los aislamientos en una misma variedad, $y$ entre variedades. Esto confirma una vez más, como ya fuera señalado por los autores (Cordo y Arriaga, 1987; Perelló et al, 1987;
Perelló et al, 1989), la presencia de especialización fisiológica en Septoria tritici.

\section{Tipos y dimensiones de conidios in vitro}

Los tipos y dimensiones de esporas de los 8 aislamientos se resumen en la tabla III. Los aislamientos $L_{5}, M_{16}$ y $D_{11}$ se caracterizaron por producir, a los $30 \mathrm{~d}$ de desarrollo en APG, picnidiosporas filiformes típicas de la especie. Los aislamientos $\mathrm{LH}_{5} \mathrm{~m}$ y $\mathrm{P}_{1}$ produjeron conidios secundarios libres más pequeños y esporas unicelulares, hialinas, redondeadas u ovoides, de $3,75-5,62 \times 3,75 \mu \mathrm{m}$, a las que se han denominando microsporas libres. Estas últimas se han visto como único tipo de esporas en el aislamiento $\mathrm{P}_{3}$.

\section{Tipos y dimensiones de conidios in vivo}

Todos los tipos culturales produjeron en ambas variedades, lesiones con picnidios, pero las esporas contenidas en ellos fueron de distinta clase (tabla III). Los aislamientos $\mathrm{B}_{18}, \mathrm{LH}_{5}, \mathrm{M}_{16}$, $D_{11}$ y $P_{6}$, produjeron picnidios con picnidiosporas típicas, tal como sucedió in vitro. Los aislamientos $P_{1}$ y $P_{3}$ produjeron picnidios que contenían sólo micropicnidiosporas, y el aislamiento $\mathrm{LH}_{5} \mathrm{~m}$, picnidios con macro y micropicnidiosporas (fig 4).

Las microsporas desarrolladas in vivo fueron mucho más pequeñas que las obtenidas en cultivo artificial $(0,5-1 \times 0,5 \mu \mathrm{m})$.

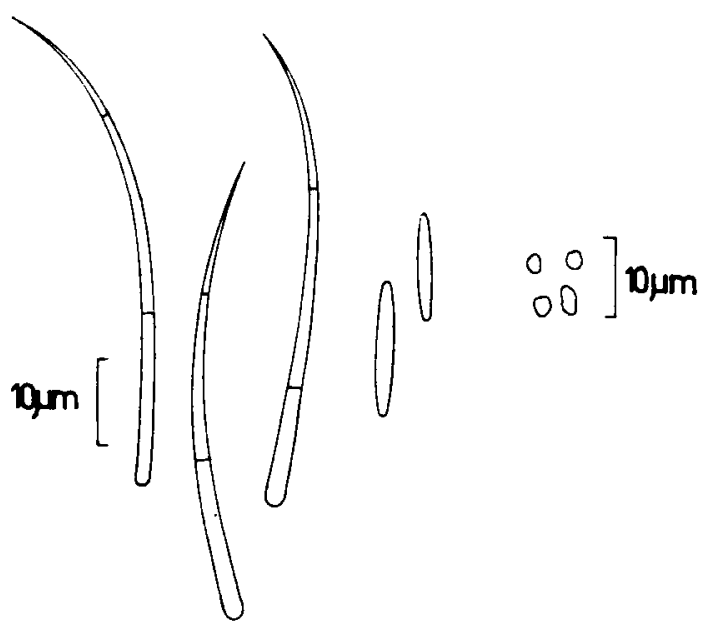

Fig 4. Cámara clara illustrando las picnidiosporas, conidios secundarios y microsporas de $S$ tritici. 
Tabla III. Dimensiones y tipos de esporas de los 8 aislamientos de $S$ tritici desarrolladas in vitro e in vivo.

\begin{tabular}{|c|c|c|c|c|c|}
\hline \multirow{2}{*}{ Aislamiento } & \multirow{2}{*}{ Tipo } & \multicolumn{2}{|r|}{ Esporas } & \multicolumn{2}{|c|}{ "In vivo" } \\
\hline & & Largo $(\mu m)$ & Ancho $(\mu m)$ & Largo $(\mu m)$ & Ancho $(\mu m)$ \\
\hline LH5 & $\begin{array}{l}\text { Picnidiosporas } \\
\text { Picnidiosporas }\end{array}$ & $\begin{array}{l}37,50-45 \\
27,50-45\end{array}$ & $\begin{array}{l}3,75 \\
3,75\end{array}$ & $\begin{array}{c}43,20-50,30 \\
36,50-75\end{array}$ & $\begin{array}{l}3,75 \\
3,75\end{array}$ \\
\hline B18 & $\begin{array}{l}\text { Conidios secundarios } \\
\text { Conidios secundarios }\end{array}$ & $\begin{array}{l}15-22,50 \\
15-22,50\end{array}$ & $\begin{array}{l}3,75 \\
3,75\end{array}$ & - & $\begin{array}{l}- \\
-\end{array}$ \\
\hline $\begin{array}{l}\text { LH5m } \\
\text { M16 } \\
\text { D11 }\end{array}$ & $\begin{array}{l}\text { Microsporas libres } \\
\text { Picnidiosporas } \\
\text { Picnidiosporas } \\
\text { Picnidiosporas }\end{array}$ & $\begin{array}{c}3,75-5,62 \\
45,50-49,20 \\
40,23-46 \\
30,20-45\end{array}$ & $\begin{array}{l}3,75 \\
3,75 \\
3,75 \\
3,75\end{array}$ & $\begin{array}{c}0,5-1 \\
48,50-52 \\
39,15-48,50 \\
34,28-49\end{array}$ & $\begin{array}{l}0,5 \\
3,75 \\
3,75 \\
3,75\end{array}$ \\
\hline P6 & $\begin{array}{l}\text { Conidios secundarios } \\
\text { Conidios secundarios } \\
\text { Microsporas libres } \\
\text { Microsporas libres }\end{array}$ & $\begin{array}{c}7,50-15 \\
3,75-5,62 \\
3,75-5,62 \\
3,75-5,62\end{array}$ & $\begin{array}{l}3,75 \\
3,75 \\
3,75 \\
3,75\end{array}$ & $\begin{array}{c}- \\
\overline{-} \\
0,5-1 \\
0,5-1\end{array}$ & $\begin{array}{l}- \\
\overline{0}, 5 \\
0,5\end{array}$ \\
\hline
\end{tabular}

* Datos promedio de 100 observaciones.

\section{DISCUSIÓN}

La producción de microsporas en $S$ tritici fue primeramente señalado por Sprague, 1950, quien las describe como similares a células bacterianas, curvadas o en forma de gancho, asietadas, hialinas, de 5-9 $\times 0.3-1 \mu \mathrm{m}$. Se indica también, que son uninucleadas (Shaw, 1953) y que fueron halladas en picnidios en la cavidad subestomática de tallos de trigo (Brokenshire, 1975). Como se ha sugerido (Brokenshire, 1975), no actúan como vía de inóculo y funcionarían como espermas en la reproducción sexual.

A diferencia de lo señalado, las microsporas descriptas en este trabajo, fueron rectas o redondeadas y más pequeñas. Se encontraron tanto en cultivo artificial como en las lesiones de hojas de trigo infectadas bajo condiciones naturales. En este último caso, se hallaron dentro de picnidios, como micropicnidiosporas, sólas o asociadas con las típicas picnidiosporas de la especie. En cultivo artificial, en ningún caso desarrollaron fructificaciones (picnidios), sino que se encontraron como microconidios libres. A través de los ensayos de inoculación se comprobó la capacidad infectiva de estas microsporas. Esto indicaría, como fuera señalado por Harrower (1976) para $S$ nodorum, que las microsporas actuarían en el mecanismo de dispersión y propagación del patógeno. La presencia de estas microsporas en $S$ tritici confirma el pleomorfismo del género Septoria, que ya fuera señalado por Johnson (1952).
Además de las diferencias en los caracteres morfobiométricos, en esta investigación se comprueba también la existencia de diversos hábitos de crecimiento en $S$ tritici. Annone (1984), para cepas de EEUU, menciona la presencia de los tipos conidial, miceliar e intermedio, con una frecuencia 4:2:1. En el país, Cordo y Lindquist (1987), definen 2 tipos de variantes culturales en $S$ tritici: miceliar y picnidial (típico), siendo este último, el más frecuente en nuestros aislamientos.

Así mismo, entre los aislamientos de $S$ tritici, se observaron variantes respecto al tipo parental, en diferentes generaciones de subcultivos monospóricos. Similares observaciones fueron realizadas por Hooker (1957) en $S$ avenae y Skajennikoff y Rapilly (1989) en S nodorum.

Con la aparición de los tipos albino, mixto y levaduroide, se reafirma que $S$ tritici es un hongo variable, capaz de producir distintas formas fisiológicas en medio artificial. Estos nuevos tipos aparecieron por aislamientos desde cultivares de trigo con germoplasma extranjero resistente de reciente introducción al país (Bobwhite "S" y sus líneas derivadas). Podría suponerse, como lo explicaron Skajennikoff $y$ Rapilly (1989) para $S$ nodorum que los distintos biotipos coexistieron siempre, pero que se manifestaron, desplegando su potencial patogénico a partir del pasaje por ciertos hospedantes selectivos (Bobwhite "S") que facilitarian su desarrollo.

Por otra parte, las pruebas de invernáculo, revelan claramente que muchos de los tipos que 
fueron diferentes culturalmente, también to fueron en su capacidad patogénica.

Desde el punto de vista del mejoramiento genético, el conocimiento de la relación entre las características culturales y patogénicas de los biotipos es de extraordinaria importancia para seleccionar las mezclas de cepas a utilizar en inoculaciones artificiales del patógeno.

Así, $S$ tritici, comprende un número de aislamientos que difieren entre ellos distintiva y consistentemente en patogenicidad, características morfobiométricas y en las características culturales sobre medio artificial.

La base genética de esta variación es difícil de interpretar. Se ha sugerido que esta variación refleja la segregación de diferentes genotipos desde heterocariones complejos originados a través de la acumulación de núcleos mutantes dentro del micelio, y del intercambio nuclear entre micelios, seguidos de una fusión hifal (Newtoy Catten, 1983).

Por último, $S$ tritici, tal como muchas otras especies de hongos fitopatógenos, es un organismo dinámico. De ahí, que comprenda una población de naturaleza cambiante, originando, aparentemente, nuevos biotipos o formas fisiológicas.

\section{AGRADECIMIENTOS}

Los autores desean agradecer a los Ing Agr JG Annone, por facilitar el resumen de su Tesis Doctoral y $S$ Sarandón, por su asistencia en el análisis estadístico de datos.

\section{REFERENCIAS}

Annone JG (1984) Cultural types of Septoria tritici Rob Ex Desm : Occurrence in Washington, stability and virulence. MS Thesis, Washington State Univ, Pullman, Washington, $68 \mathrm{p}$

Annone JG (1988) Obsenvación de conidios secundarios de Septoria tritici por medio de impresiones foliares. Informe técnico $\mathrm{n}^{\circ} 214$ INTA. Instituto Nacional de Tecnología Agropecuaria Pergamino, Rca Argentina

Brokenshire T (1974) Studies on Septoria tritici Desm Ph D Thesis, Univ Exeter

Brokenshire T (1975) The role of Graminaceous species in the epidemiology of Septoria tritici on wheat. Plant Pathol 24, 33-38

Commonwealth mycological institute (1985) Medios de cultivo y métodos micológicos. In: Manual para patólogos vegetales $\mathrm{FAO}, \mathrm{CMI}, \mathrm{CAB}$
Cordo C, Arriaga HO (1987) Variation in pathogenicity among strains of Mycosphaerella graminicola (anamorph Septoria tritici). In: Proc of the Taller sobre avances y Metodología sobre Septoriosis en Cereales. CIMMYT MAP IPO México. Noviembre 9-14 1987 La Estanzuela, Uruguay (in press)

Cordo C, Lindquist J (1987) Análisis cualitativo de la variabilidad cultural de Septoria tritici. Bol Soc Argent Bot 25, 59-77

Dickson JG (1947) Diseases of Field Crops. Mc Graw Hill Book, Inc, New York, London, 429 p

Djerbi M (1972) Contribution à l'étude de la sporulation du Septoria tritici Desm. Arch Inst Pasteur Tunis, $49,61-68$

Eyal Z, Scharen AL, Prescott JM, Van Ginkel M (1987) Enfermedades del trigo causadas por Septoria: Conceptos y métodos relacionados con el manejo de estas enfermedades. México, DF: CIMMIT

Gerassini LA (1958) Microbiologia (1 edición) Univ Cent Venezuela. Sucre, 230-233

Harrower KM (1976) The micropycnidiospores of Leptosphaeria nodorum. Trans Br Mycol Soc 67, 33-38

Hooker AL (1957) Cultural variability in Septoria avenae through successive single macrospore transfers. Phytopathology 47, 460-468

Jonhson T (1952) Cultural variability in Septoria avenae Frank. Can J Bot 10, 318-332

Jones GD, Lee N (1974) Production of secondary conidia by Septoria tritici in culture. Trans $\mathrm{Br} M y c o l$ Soc 62, 212-214

Negroni P (1938) Morfología y Biología de los Hongos. Técnicas Micológicas. El Ateneo Bs Aires $299 \mathrm{p}$

Newton AC, Catten CE (1983) Heterocaryosis and heterocaryon incompatibility in Septoria nodorum. Proc Workshop Held August 2-4 at Montana State Univ 13-14

Perelló A, Babinec F, Cordo C (1987) Proc of the Taller sobre avances y metología sobre Septoriosis en Cereales CIMMYT/MAP/IPO México Noviembre 9141987 La Estanzuela Uruguay (in press)

Perelló A, Cordo C, Arriaga HO, Alippi H (1989) Variation in virulence in isolates of Septoria tritici Rob Ex Desm on wheat. In: Proc of the Third International Workshop on Septoria Diseases of Cereals, July 47, Swiss Fed Res St Agron, Zurich, Switzerland, 43-46

Rayner RW (1970) A mycological colour chart. Common Mycol Inst. Kew, Surrey and Brit Mycol Soc

Shaw DE (1953) Cytology of Septoria and Selenophoma spores. Proc Linn Soc N S W 78, 122-130

Skajennikoff MF, Rapilly (1983) Études sur l'agressivité de Septoria nodorum Berk. Effets des hôtes (triticale et blé) et des organes attaqués. Agronomie 3, 131-140

Skajennikoff MF, Rapilly (1989) Variabilité du pouvoir pathogène chez Septoria nodorum Berk (Leptosphaeria nodorum Müll) Agronomie 9, 693-702

Sprague R (1950) Diseases of cereals and grasses in North America. The Ronald Press Co, New York, $538 p$ 